\title{
Characterization of a Piezoelectric Inkjet Aerosol Generator for the Study of Bioaerosol Survivability
}

\author{
Chih-Wei Lin ${ }^{1}$, Ting-Ho Kuo ${ }^{1}$, Sheng-Hsiu Huang ${ }^{1}$, Yu-Mei Kuo ${ }^{2 *}$, Wen-Jong Wu ${ }^{3}$, \\ Chih-Chieh Chen ${ }^{1 * *}$
}

${ }^{1}$ Institute of Occupational Medicine and Industrial Hygiene, College of Public Health, National Taiwan University, Taipei 10055, Taiwan

${ }^{2}$ Department of Occupational Safety and Health, Chung Hwa University of Medical Technology, Tainan 71703, Taiwan

${ }^{3}$ Department of Engineering Science and Ocean Engineering, National Taiwan University, Taipei 10617, Taiwan

\begin{abstract}
In laboratory-based bioaerosol researches, the ability to reliably generate bioaerosols with minimal damage to the microorganisms is critical. In this work, a thermally consistent inkjet-based aerosol generation technique was adopted to generate bioaerosols, and its ability to preserve microbial survivability was assessed. The performance of the piezoelectric inkjet aerosol generator was evaluated using a potassium sodium tartrate solution and an aqueous suspension of 3- $\mu \mathrm{m}$ polystyrene latex spheres. An Aerodynamic Particle Sizer was employed to measure the aerosol number concentration and the size distribution. The main operating parameters of the inkjet print head for generating viable aerosols were the driving voltage, pulse duration, dispersion air flow, pulse frequency, and volume fraction of the solids in the nebulizing liquid. In the bioaerosol survivability experiments, yeast was used as the test microorganism. The nebulized yeast cells were sampled at different distances from the outlet of the inkjet aerosol generator and thus exposed to varying degrees of relative humidity for different residence times. Three bioaerosol samplers, namely, a BioSampler, a gelatin filter cassette, and a Marple cascade impactor with Mylar film, were used to collect the airborne yeast samples. Yeast survivability was determined by methylene blue viability staining. This study successfully demonstrated the feasibility and the advantages of using a piezoelectric inkjet print head to generate viable aerosols. Applying a square wave with appropriate combinations of voltage and pulse duration enabled the inkjet system to produce a liquid with rheological properties similar to those of water, and the particle generation rate was manipulated by adjusting the pulse frequency. Moreover, dispersion air was required to reduce coagulation. Although the number of droplets containing PSL spheres increased with the volume fraction of the spheres $\left(F_{v}\right)$, a high $F_{v}$ also reduced the probability of singlets forming.
\end{abstract}

Keywords: Survivability; Piezoelectric inkjet; Aerosol generation; Bioaerosols.

\section{INTRODUCTION}

Airborne infectious agents have received much more attention after the epidemic outbreak of the severe acute respiratory syndrome (SARS) in 2003, and airborne infectious diseases like influenza and tuberculosis are still critical issues to public health. Pathogens can be aerosolized by an infected person via coughing, sneezing, talking, and even just breathing. From the viewpoint of infection control,

\footnotetext{
* Corresponding author.

Tel.: 886-912017249; Fax: 886-6-2894028

E-mail address: ymkuo@mail.hwai.edu.tw

** Corresponding author.

Tel.: 886-928253663; Fax: 886-2-23938631

E-mail address: ccchen@ntu.edu.tw
}

the survival of an airborne pathogen in the environment plays an important role. Furthermore, bioaerosols can remain airborne for a long period of time depending on the size and are therefore widely spread. In the past decades, there have been numerous studies focusing on viable aerosol generation, sampling and control (Reponen et al., 1997; Lever et al., 2000; Mainelis et al., 2005; Wu and Yao, 2010; Tolchinsky et al., 2011; Roux et al., 2013; Zhen et al., 2014; Zuo et al., 2014; Sung et al., 2018). For laboratorybased bioaerosol studies, an aerosol generator which is less detrimental to the survivability of microorganisms is needed, especially for sensitive species.

Two nebulization methods, the pneumatic technique and ultrasonic technique, are often applied to generate droplet aerosols in a variety of research settings and applications. Based on the principle of pneumatic nebulization, the Collison nebulizer is probably the most commonly used bioaerosol generator in bioaerosol research (Reponen et 
al., 1997; Agranovski et al., 2005; Zhen et al., 2013; Zuo et al., 2014). Nevertheless, the quality of the bioaerosols generated by the Collision nebulizer has been questioned since the high-speed jet flow and solid wall impingement may exert a strong stress on microbial cells. The Collison nebulizer has been shown to injure microorganisms due to impaction and shear forces (Reponen et al., 1997; Thomas et al., 2011). In addition, the fluid is recirculated every 6 seconds in the glass reservoir containing $20 \mathrm{~mL}$ liquid as the collection solution. Microbial viability can be affected by frequent recirculation of the cell suspension and the concentration of suspension under prolonged nebulization can be changed due to the evaporation of the solvent (May, 1973).

With a special focus on minimizing damage to microorganisms, several new generators have been developed for bioaerosol research, for example, a flow-focusing aerosol generator (FFAG; Ingeniatrics Technologías, Seville, Spain) (Thomas et al., 2009) and a C-Flow concentric nebulizer (Savillex Inc., Eden, Prairie, MN, USA). With similar design based on the pneumatic technique, aerosol particles are produced by the breakup of generated microjets which are driven by the suction of a highly accelerated co-flow gas stream (Gañán-Calvo and Barrero, 1999). Other than the pneumatic technique, a single-pass bubbling generator was developed based on the bubbling technique called Liquid Sparging Aerosolizer (LSA) (Mainelis et al., 2005). All generators mentioned above use air to nebulize aerosol particles. It is sometimes difficult to generate high output of viable aerosols without increasing air flow or manipulating the characteristics of generated aerosols (for example, the amount of microbial cells contained in an aerosol and the surface coating).

In addition to frequently used pneumatic nebulizers, ultrasonic nebulizers have been also used to aerosolize viable microorganisms (Meier, 2006; Woo et al., 2012; Roux et al., 2013). However, ultrasonic nebulizers are frequently used to generate droplet aerosols for inhalation studies (Kreyling and Ferron, 1982; Lahde et al., 2006; Lin et al., 2011; Tsai et al., 2013). The increase of the temperature of the solution in an ultrasonic nebulizer is attributed to the energy generated from the piezoelectric crystal during prolonged nebulization. This may cause the evaporation of the solvent and therefore the increase of solution concentration (Porstendörfer et al., 1977). In fact, ultrasonic disinfection has been proven to be effective for killing bacteria such as E. coli, Pseudomonas aeruginosa, and Enterococcus avium (Stamper et al., 2008). In other words, ultrasonic nebulization of viable microorganisms may have a detrimental effect on microbial survivability. Moreover, ultrasonic nebulizers have been demonstrated to degrade heat-sensitive materials (Taylor and McCallion, 1997; Watts et al., 2008) and have difficulty in the aerosolization of particle suspensions (Nikander et al., 1999; Terzano and Allegra, 2002).

With controllable and reproducible droplet generation, inkjet printing has been used in fields outside its traditional use of printing. The most common use is the piezoelectric drop-on-demand (DOD) method. The print head consists of a fluid-filled glass capillary bonded to an annular piezoelectric actuator. The droplet size in terms of droplet volume is governed by the characteristics of the inkjet nozzles and rheological properties of the inkjet solutions. The outlet diameter of the nozzle is typically $20-70 \mu \mathrm{m}$ and the resulting droplets are in the size range of 10-100 $\mu \mathrm{m}$ (Reis et al., 2005). When using a piezoelectric inkjet print head to generate aerosols, it has been demonstrated that the nozzle diameter must be at least three times the particle diameter for stable particle ejection (Yamaguchi et al., 2012a). Liquid properties such as viscosity, surface tension, and density have great influence on droplet formation. Generally, a DOD mode inkjet print head is capable of nebulizing liquid with a viscosity of $1-10 \mathrm{cP}$ and a surface tension of 20-70 dyne $\mathrm{cm}^{-1}$ (Shimoda et al., 2003). Low viscosities usually lead to satellite formation. High surface tension can prevent the formation of satellite droplets (Kwon, 2010).

In addition to inkjet nozzles and nebulizing solutions, droplet formation is significantly affected by the operating conditions of the piezoelectric actuator, such as driving voltage, pulse width, and waveform. The voltage is applied to the piezoelectric actuator and induces a pressure on a squeeze type piezoelectric print head. The voltage has to be high enough to overcome the surface tension and viscous force of the nebulizing liquid and squeeze out a droplet from the inkjet nozzle. As the pulse voltage increases, both the velocity and the volume of each droplet become larger (Lin, 2006; Shih et al., 2015). However, this can also lead to the formation of satellite droplets (Kwon, 2010).

A simple trapezoidal waveform has been widely used in practice for controlling jetting behavior (Kwon, 2009). For this waveform, a fixed value is used for both the rising time and the falling time. The dwell time can be adjusted to suppress the satellite effect. However, a shorter dwell time (in terms of pulse width) may result in no droplet formation or require increased pulse voltage. After testing for three types of signal waveforms (single, double and bipolar waveform), Liu et al. (2013) concluded that a double waveform with an appropriate interval time between two pulses can generate a single droplet by creating additional negative pressure to avoid satellite droplets. Moreover, a bipolar waveform allows a high voltage difference without the application of excessively high voltage and can generate a single droplet for high viscosity fluid (Liu et al., 2013).

Commonly used bioaerosol generation methods, such as pneumatic nebulization and ultrasonic nebulization, are known to affect the viability of microorganisms. Piezoelectric inkjet printing is a thermally consistent process and therefore the viability of aerosolized cells is expected to be well preserved. Numerous studies have used piezoelectric inkjet print heads to eject tissue cells (Saunders et al., 2008; Parsa et al., 2010; Huang et al., 2012; Yamaguchi et al., 2012b; Ferris et al., 2013; The et al., 2013) or microbeads (Yamaguchi et al., 2012a; Shih et al., 2015). One study utilized piezoelectric inkjet array print heads with $30-\mu \mathrm{m}$ nozzles to nebulize 1- $\mu \mathrm{m}$ PSL spheres and bacteria spores (Dougherty et al., 2007). The experimental results show that the lower the concentration of PSL in the suspension solution, the smaller the resulting dried particles. For low 
concentrations, the highest count of aerosol particles was composed of single PSL. At higher concentrations, clusters became evident. In accordance with the appearance of the clusters observed under an electronic microscope, a concentration of 90-100 spores per 30- $\mu$ m-diameter droplet was suggested for the suspension of $6.5 \times 10^{9} \# \mathrm{~mL}^{-1}$ Bacillus globigii spores.

As a reliable viable aerosol generator, the piezoelectric inkjet print head can also manipulate the surface coating of bioaerosols by altering the rheological properties of the nebulized fluid to mimic the bioaerosols in a real-world scenario. Generally, the infectious agents produced by an index case may become airborne with saliva or mucus coating, which may affect microbial survivability. The presence of surrounding organic materials (e.g., saliva and mucus) may protect airborne pathogens as they encounter harsh environmental conditions like dehydration, ultraviolet radiation (Woo et al., 2012) and so forth. A laboratory-based study found that a virus carried by larger particles may be surrounded by more organics (e.g., solutes in the nebulizer suspension) as compared with a virus existing as a singlet or in association with fewer organic materials (Zuo et al., 2014). As a result, virus carried by larger particles exhibited higher survivability. It has been speculated that the surface coating materials provide a shielding effect for microorganism survivability and may also reduce sampling stress such as desiccation and sampler dependent-mechanical forces.

Focusing on infectious bioaerosols, previous studies have often used commercial or home-made nebulizers to generate polydisperse bioaerosols. However, the generated droplets may contain more than one microbial cell and form cell clusters with surface coating when dried. Varying characteristics of challenging viable aerosols may bias the effectiveness of infectious aerosol control measures in practical applications. Moreover, it is sometimes difficult to make generalizations. Few studies have described the generation of viable aerosols, especially with respect to the likelihood of multiplets and surface coating as well as their impact on microbial viability. In the present study, a piezoelectric inkjet print head was used to generate bioaerosols. The characteristics of the resulting bioaerosols were manipulated by adjusting the concentration of microbial suspension and the liquid properties. The objectives of this work were to characterize a piezoelectric inkjet system for generating viable aerosols with given characteristics and to set up a settling chamber with controllable airborne time for the study of bioaerosol survivability.

\section{MATERIALS AND METHODS}

\section{Inkjet Aerosol Generation System}

The aerosol generation system, as shown in Fig. 1, consisted of an inkjet print head (MJ-AT-50, MicroFab Technologies Inc., Plano, Texas, USA), a function generator, a power amplifier, and a pressure control device. A squeeze mode piezoelectric print head with a 50 - $\mu$ m-diameter nozzle was used in the present study. It consisted of a glass capillary bonded to an annular piezoelectric actuator, for bipolar pulse excitation. This inkjet print head could be used to atomize liquid suspension with viscosity less than $20 \mathrm{cP}$ and surface tension between 20 to 70 dyne $\mathrm{cm}^{-1}$, which is similar to water.

The piezoelectric inkjet print head was driven by a series of square pulses produced from a function generator. The signal was acquired by a data acquisition card (DAQ card, NI PCI-6251, National Instruments Co., Austin, TX, USA) with the maximal output of $10 \mathrm{~V}$. A power amplifier (HA-400, PINTEK Co., Ltd., Taipei, Taiwan) was employed to amplify the output signal up to $100 \mathrm{~V}$. The signal waveform was controlled using a LabVIEW program (LabVIEW 2011, National Instruments Co.) and the rising and falling times for a square wave pulse were both less than $100 \mathrm{~ns}$. To visualize the droplet formation and print head clog, a charge-coupled device (CCD, SuperIris-360CA, New Image Co., Ltd., Taipei, Taiwan) and a strobe LED were used. The CCD achieved a frame rate of up to

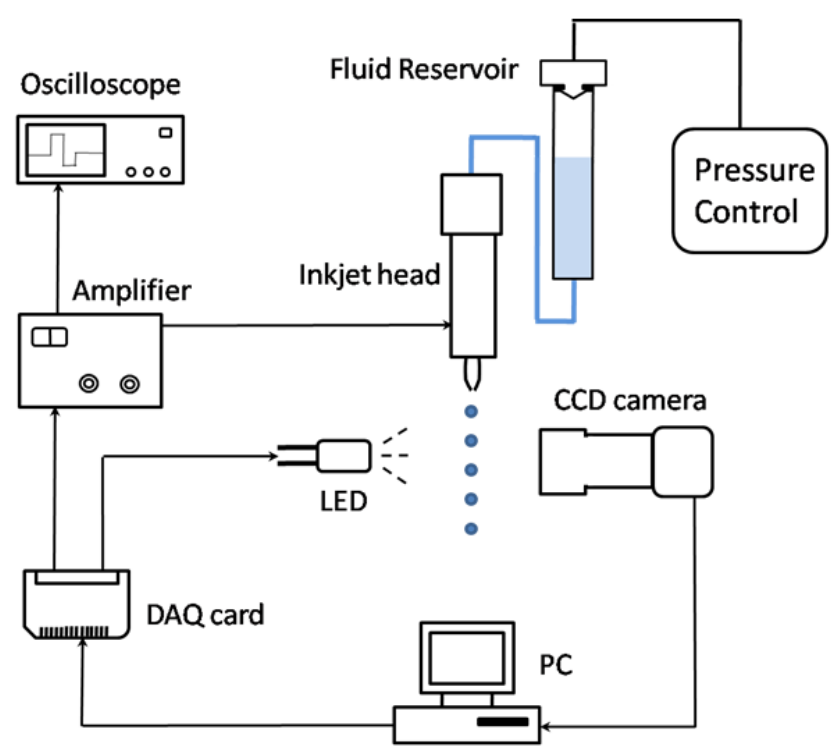

Fig. 1. Schematic diagram of the inkjet system. 
30 frames per second (fps) with a resolution of $1280 \times 960$ pixels. The LED was operated at a frequency of $1000 \mathrm{~Hz}$ and exposure time of $1 \mu \mathrm{s}$.

\section{Characterization of Inkjet Aerosol Generator with Non-Biological Particles}

The performance of the aerosol generation system was characterized using potassium sodium tartrate solution (PST) and 3- $\mu \mathrm{m}$ polystyrene latex (PSL) suspension. The PST solution was used to evaluate the characteristics of droplets generated by an inkjet print head with a $50-\mu \mathrm{m}-$ diameter nozzle. The solubility and density of PST (MW 282) in water is $0.63 \mathrm{~g} \mathrm{~mL}^{-1}$ and $1.79 \mathrm{~g} \mathrm{~cm}^{-3}$. The $3-\mu \mathrm{m}$ PSL was used as a surrogate in this case because the size is close to microorganisms such as yeast.

The operating parameters including driving voltage, pulse width, dispersion air flow rate, pulse frequency and volume fraction of solids in the suspension were evaluated. To prevent the formation of satellite droplets, bipolar square pulses were generated to drive the inkjet print head and the time needed for a square pulse to rise and fall was less than $100 \mathrm{~ns}$. The test driving voltage and pulse width varied from 10 to $55 \mathrm{~V}$ and from 6 to $23 \mu \mathrm{s}$, respectively. The particle concentration measurements were conducted with dispersion air supply varying from 0 to $1 \mathrm{~L} \mathrm{~min}^{-1}$ in increments of $0.2 \mathrm{~L} \mathrm{~min}^{-1}$. The particle generation rate is solely controlled by the frequency of voltage pulses sent to the inkjet print head and a drop-on-demand inkjet print head typically generates droplets at a rate in the range of $0.1-10 \mathrm{kHz}$ (Reis et al., 2005). Based on the preliminary observation, the inkjet printing head cannot properly generate droplets with uniform size at a pulse frequency higher than $9000 \mathrm{~Hz}$. Therefore, in this work, the pulse frequency was set to vary from 10 to $7000 \mathrm{~Hz}$ to suppress the formation of satellite droplets. Under the studied pulse frequency range, only one droplet was generated each time as the single electrical pulse was applied to the inkjet print head. The droplet generation rate can be estimated by the applied pulse frequency. Subsequently, the aerosol number concentration can be predicted by the following equation.

$$
C=\frac{\text { pulse frequency }}{Q_{T}}
$$

where $\mathrm{Q}_{\mathrm{T}}$ is the total air flow rate. In this work, $\mathrm{Q}_{\mathrm{T}}$ was 6.0 $\mathrm{L} \mathrm{min}^{-1}$, unless otherwise specified.

The test PSL suspension concentration varied from $5 \times$ $10^{5}$ to $5 \times 10^{7}$ particles $\mathrm{mL}^{-1}$. The fluid reservoir was pressurized negatively to overcome any capillary dripping under idle mode. Under jetting mode, a Y-connector attached to a vacuum pump and a compressed air supply fed negative and positive pressure to the fluid reservoir and back pressure was adjusted to provide stable droplet generation. An Aerodynamic Particle Sizer (APS; Model 3321, TSI Inc., St. Paul, MN, USA) was employed to measure the aerosol number concentration and size distribution in the range of $0.5-20 \mu \mathrm{m}$.

\section{Preparation of Yeast Suspension for Nebulization}

The yeast survivability study was performed using a settling chamber, as shown in Fig. 2. The yeast (Saccharomyces cerevisiae) was chosen due to the low health risk, easy cultivation and short generation time. The yeast was first cultivated on yeast extract peptone dextrose (YPD) medium. After overnight incubation at $30^{\circ} \mathrm{C}$, a single colony was picked and inoculated into $10 \mathrm{~mL}$ YPD broth. The broth cultures were incubated in a shaking water bath at $100 \mathrm{rpm}$, for $18-24$ hours at $30^{\circ} \mathrm{C}$. The yeast broth was centrifuged at $4000 \mathrm{rpm}$ for 10 minutes to remove the broth. Then the cell pellet was re-suspended and washed with disinfected water twice. The concentration of the yeast

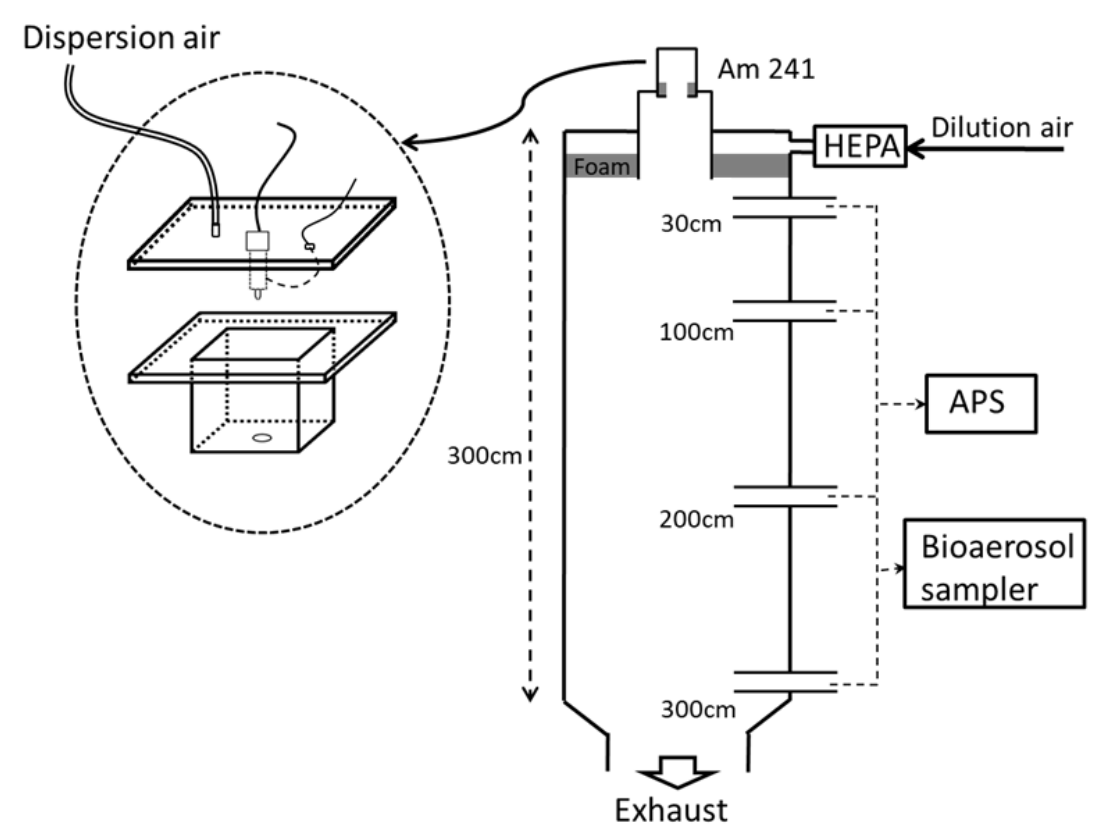

Fig. 2. The settling chamber for the yeast survivability test. 
suspension was measured using a counting chamber. The yeast suspension was then diluted with disinfected water to achieve a desired concentration and then introduced into the fluid reservoir for nebulization.

\section{Performance Evaluation of an Inkjet Aerosol Generator for Yeast Survivability}

To rate the performance of the piezoelectric inkjet print head in the generation of viable yeast for survivability test, the piezoelectric inkjet and two types of nebulizers, including a six-jet Collison nebulizer (Model CN25, BGI Inc., Waltham, MA, USA) and an ultrasonic nebulizer (ultrasonic atomizing nozzle; 8700-120 MicroSpray, SonoTek Inc., Milton, NY, USA), were evaluated. The freshly nebulized aerosol particles were sprayed on a clean glass slide and subsequently stained with $0.01 \%$ methylene blue for 10 minutes. Several hundreds of cells were enumerated under a light microscope at $400 \times$ magnification and the percentage of cell survivability (survival rate) was calculated by dividing the number of unstained cells by the total number of cells counted. The relative survival rate, defined as the survival rate ratio of the nebulized sample to the suspension, was used to describe the effect of nebulization methods on yeast survivability.

\section{Performance Comparison of Bioaerosol Sampling Methods}

Driven by an appropriate voltage-pulse signal, the inkjet print head was employed to generate droplets containing yeasts and dry filtered air flew around the nozzle outlet for aerosol dispersion. After being neutralized by a radioactive source, Am-241, and diluted with filtered air, the aerosol output was introduced into a 3-m high vertical acrylic cylinder (diameter: $20 \mathrm{~cm}$ ) to expose it to varying relative humidities (20-80\% RH). Subsequently, airborne yeast samples were collected at different distances $(30,100,200$, and $300 \mathrm{~cm}$ ) from the outlet of inkjet nozzle to evaluate the effect of residence time on yeast survivability. Three types of bioaerosol samplers, including a BioSampler (SKC, Inc., Eighty Four, PA, USA), a filter cassette with a $25-\mathrm{mm}$ gelatin filter (SKC, Inc., Eighty Four, PA) and a Marple cascade impactor (Model 290, Tisch Environmental, Inc., Cleves, OH, USA) with Mylar film, were used to collect yeast aerosols. The SKC BioSampler using water as collection liquid operated at $12.5 \mathrm{~L} \mathrm{~min}^{-1}$ for 15 minutes. However, the flow rate of the BioSampler exceeded the air supply inside the settling chamber. To overcome this problem, bypass air of $10.5 \mathrm{~L} \mathrm{~min}^{-1}$ was re-circulated and only $2 \mathrm{~L} \mathrm{~min}^{-1}$ of air was sampled directly from the test chamber. After sampling, the yeast cells in the collection liquid were concentrated by centrifuging at $4000 \mathrm{rpm}$ for 10 minutes. The gelatin filter cassette was operated at $2 \mathrm{~L} \mathrm{~min}^{-1}$ for 6 minutes. After sampling, disinfected water was added to dissolve the gelatin filter and make a yeast suspension. In both cases, an aliquot of the extracted yeast suspension was mixed with methylene blue solution for viable staining. The Marple cascade impactor with Mylar film was operated at $2 \mathrm{~L} \mathrm{~min}^{-1}$ for 3 minutes and the staining solution was directly applied onto the collection substrate after sampling. The relative survival rate (RSR) was used to describe the impact on yeast survivability and was given by:

$$
R S R=\frac{S R_{\text {extract }}}{S R_{\text {nebulizer }}} \text { or } R S R=\frac{S R_{\text {filter }}}{S R_{\text {nebulizer }}}
$$

where $S R_{\text {extract }}$ represents the yeast survival rate of an extracted suspension with a BioSampler or a gelatin filter cassette, $S R_{\text {nebulizer }}$ represents the survival rate of the freshly nebulized sample collected by a glass slide, and $S R_{\text {filter }}$ represents the yeast survival rate of a filter sample with a Marple cascade impactor and Mylar film.

\section{RESULTS AND DISCUSSION}

\section{Operating Conditions for the Inkjet Aerosol Generator}

The piezoelectric inkjet print head utilized a piezoelectric actuator to generate high positive pressure for forcing a certain amount of liquid out of the fluid reservoir through the nozzle. When the pressure overcame the restoring effects of surface tension and viscous force, the liquid ligament was broken up, followed by droplet formation. Based on visual observation with water as the test liquid, it was found that liquid could be held in position inside the glass capillary when back pressure was regulated in the range of -1.8 to $-3.4 \mathrm{kPa}$ under jetting mode. Beyond that range, either the liquid would drain from the nozzle tip of the glass capillary or the droplet would not be generated.

To determine the operating conditions for generating monodisperse droplets, the function generator produced a series of bipolar square pulses with amplitudes varying from 12 to $55 \mathrm{~V}$ and pulse width ranging from 6 to $23 \mu \mathrm{s}$. Water was used to generate droplets at $16 \% \mathrm{RH}$ and $27^{\circ} \mathrm{C}$. The back pressure was maintained at $-2.2 \mathrm{kPa}$. The inkjet print head did not eject droplets until the pulse voltage was over $12 \mathrm{~V}$. The pulse width was adjusted to ensure the generation of single droplets free of satellites. The working range was determined using the maximum and minimum values of observations. As a result, monodisperse droplets could only be produced under a limited range of combinations of voltage and pulse width, as shown in the dark shaded area in Fig. 3.

\section{Effect of Dispersion Air on Particle Generation}

For each combination of voltage and pulse width, the inkjet print head produced monodisperse droplets. However, it was observed that the particle concentration did not increase as the excitation frequency increased from 500 to $1000 \mathrm{~Hz}$ when using PST as the nebulizing solution. It is speculated that the formed droplets began to coagulate in the vicinity of the nozzle opening due to an overwhelming increase in the number of droplets right at the exit of the nozzle. To prevent droplet coagulation, which would induce a broadening of particle size distribution, dispersion air was applied to the nozzle opening, as shown in Fig. 2. The effect of dispersion air on the particle concentration was evaluated with PST solution at two excitation frequencies, 500 and $1000 \mathrm{~Hz}$, as shown in Fig. 4. The studied dispersion 


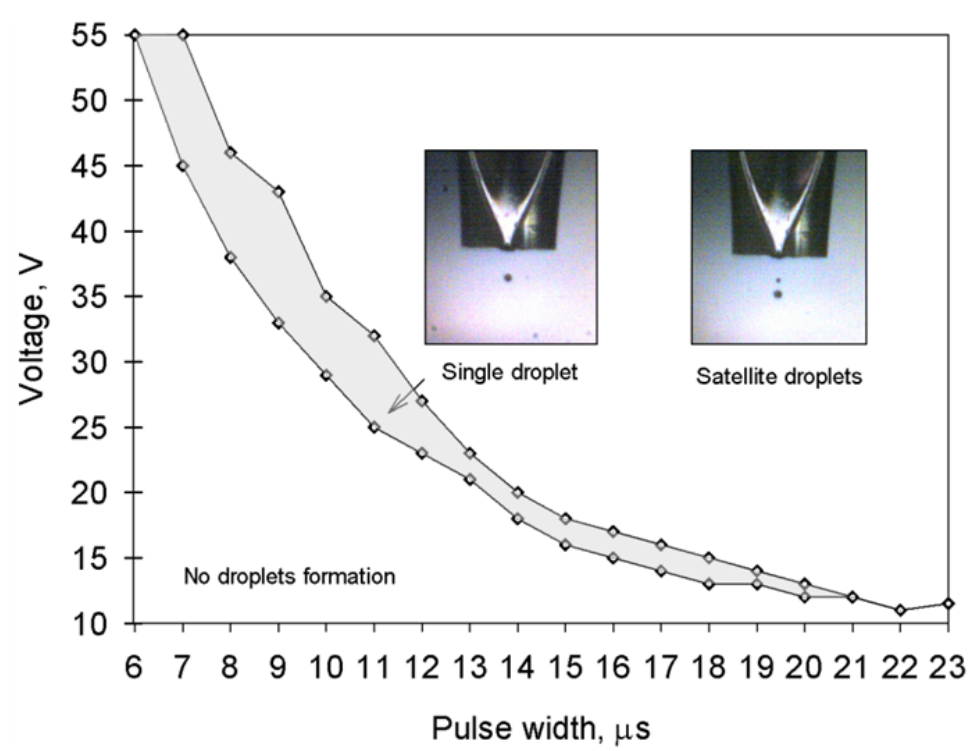

Fig. 3. The working range of the inkjet print head.

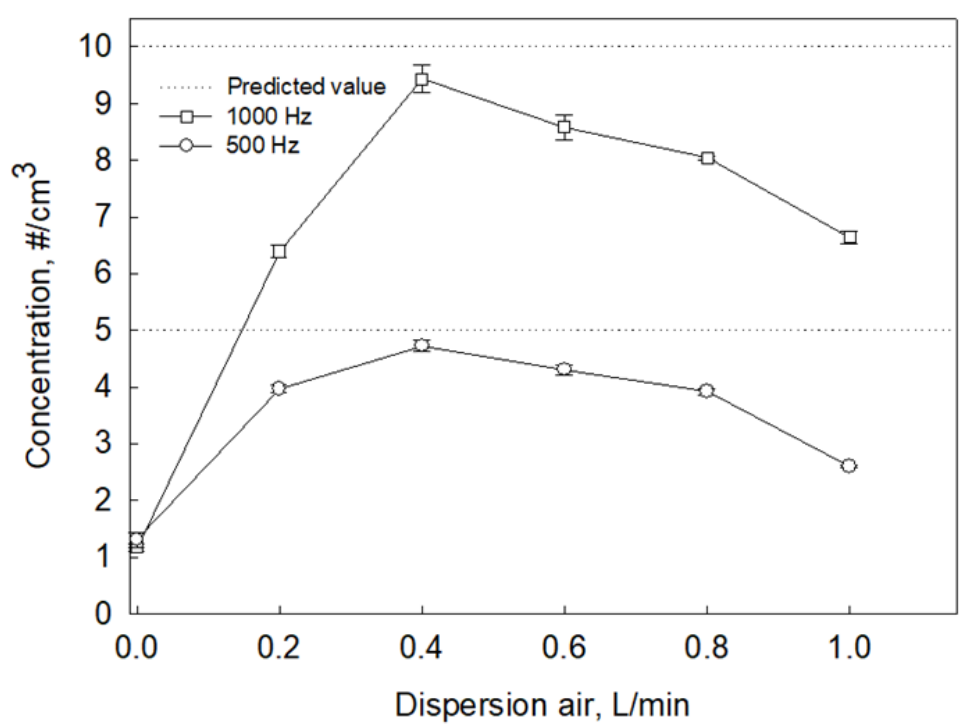

Fig. 4. Effect of dispersion air on the aerosol concentration.

air flow varied from 0 to $1 \mathrm{~L} \mathrm{~min}^{-1}$ at an increment of $0.2 \mathrm{~L} \mathrm{~min}^{-1}$ with the overall air flow of $6 \mathrm{~L} \mathrm{~min}^{-1}$, including the dilution air flow. Particle generation efficiency is defined as the number of aerosol particles generated by one voltage pulse sent to an inkjet head (Iida et al., 2014). For aerosol sizes ranging from 0.5 to $20 \mu \mathrm{m}$, the aerosol generation efficiencies at different dispersion air flows were listed in Table 1. The measured aerosol concentration was far below the theoretical value when there was no dispersion air supply. However, the measured aerosol concentration gradually approached the theoretical value and achieved a maximum level as the dispersion air flow increased to $0.4 \mathrm{~L} \mathrm{~min}^{-1}$. The measured aerosol concentration began to decline when the dispersion air was over $0.4 \mathrm{~L} \mathrm{~min}^{-1}$. This might be attributed to the increasing aerosol impaction on the inner surface of the chamber due to turbulence caused by increasing dispersion air.

\section{Effect of Pulse Frequency on Particle Generation}

Fig. 5 shows the deviation of measured aerosol concentration from the predicted value, with and without dispersion air at different excitation frequencies, including 50, 100, 250, 500 and $1000 \mathrm{~Hz}$. The results show that the particle concentration was gradually underestimated as the excitation frequency increased, very likely due to aerosol coagulation. This phenomenon was particularly significant when no dispersion air was supplied.

With dispersion air fixed at $0.4 \mathrm{~L} \mathrm{~min}^{-1}$, Fig. 6 shows the performance of the inkjet print head under different excitation frequencies, including 500, 1000, 3000 and $7000 \mathrm{~Hz}$. A $0.0216 \%$ solution of PST in water was used. The operating voltage and pulse width were $16 \mathrm{~V}$ and 16 $\mu \mathrm{s}$, respectively. The back pressure was maintained at -2.2 $\mathrm{kPa}$. The total flow rate in the chamber was $6 \mathrm{~L} \mathrm{~min}^{-1}$. Experimental results showed that, when sampled from the 
Table 1. Effect of dispersion air on particle generation efficiency.

\begin{tabular}{lllllll}
\hline \multirow{2}{*}{ Frequency, $\mathrm{Hz}$} & \multicolumn{5}{c}{ Dispersion air flow rate, $\mathrm{L} \mathrm{min}^{-1}$} \\
\cline { 2 - 7 } & 0 & 0.2 & 0.4 & 0.6 & 0.8 & 1.0 \\
\hline 500 & $0.26 \pm 0.03$ & $0.79 \pm 0.01$ & $0.95 \pm 0.02$ & $0.86 \pm 0.02$ & $0.78 \pm 0.01$ & $0.52 \pm 0.01$ \\
1000 & $0.12 \pm 0.01$ & $0.64 \pm 0.01$ & $0.94 \pm 0.02$ & $0.86 \pm 0.02$ & $0.80 \pm 0.01$ & $0.66 \pm 0.01$ \\
\hline
\end{tabular}

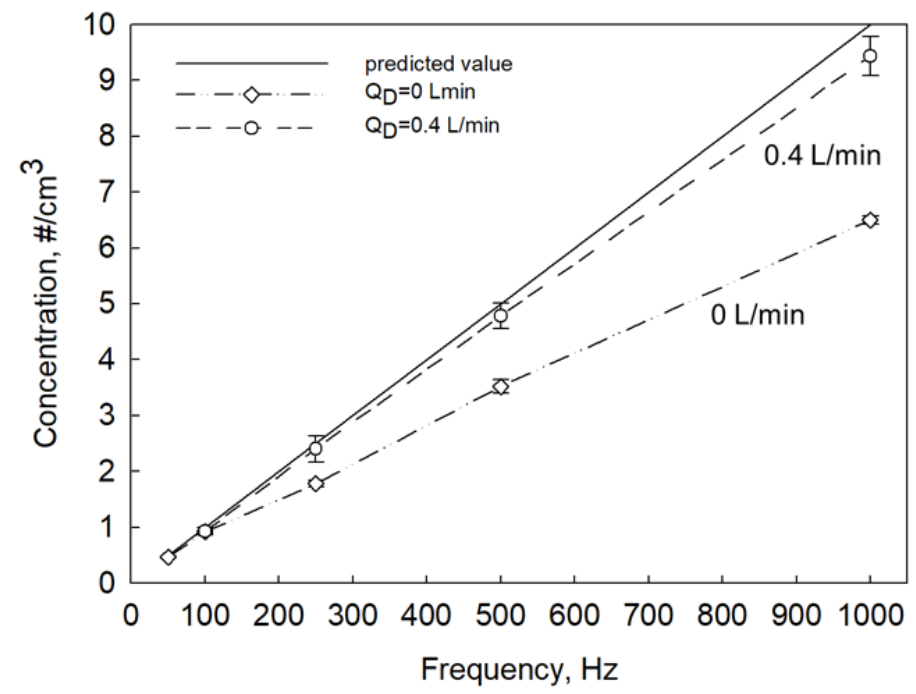

Fig. 5. Impact of dispersion air on the aerosol concentration at different excitation frequencies.

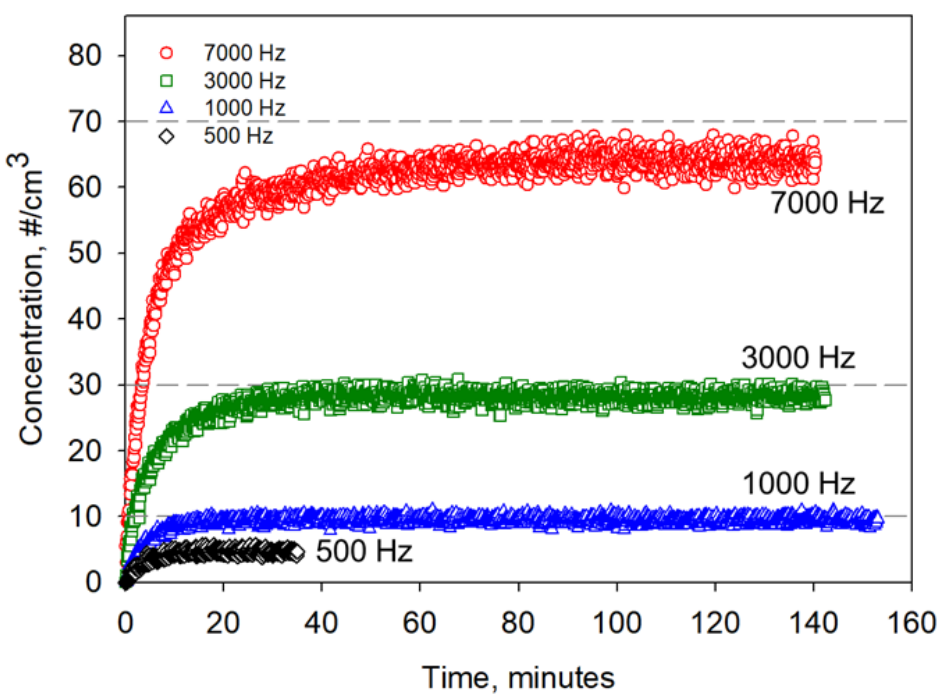

Fig. 6. Performance of the inkjet print head under different frequencies.

bottom of the 3-m vertical acrylic cylinder, the time required to reach stable concentration was approximately 25 minutes. Moreover, the equilibrium aerosol concentration in the mixing chamber at 500, 1000, 3000, and $7000 \mathrm{~Hz}$ reached $94,95,94$, and $92 \%$ of the theoretical value, respectively. High frequency of $7000 \mathrm{~Hz}$ presented the lowest fraction of the predicted concentration, likely due to aerosol coagulation.

\section{Nebulization of PST Solution by Inkjet Aerosol Generator}

In theory, the size of the final aerosol particle $\left(d_{p}\right)$ depends on the volume fraction of solute material $\left(F_{v}\right)$ and the droplet diameter $\left(d_{d}\right)$ is given by (Hinds, 1999):
$d_{p}=d_{d}\left(F_{v}\right)^{1 / 3}$

To evaluate the size of droplets generated by the inkjet print head with a $50-\mu \mathrm{m}$-diameter nozzle, three combinations of voltage and pulse width $(14 \mathrm{~V}-18 \mu \mathrm{s}, 16 \mathrm{~V}-16 \mu \mathrm{s}$ and $18 \mathrm{~V}-14 \mu \mathrm{s})$ were randomly chosen to generate monodisperse droplets based on the working range of the inkjet print head. A $0.0216 \%$ solution of PST in water was nebulized to produce solid particles of the desired size. The back pressure was kept at $-2.2 \mathrm{kPa}$ and the excitation frequency was $500 \mathrm{~Hz}$. The flow rates of dispersion air and dilution air were 0.4 and $5.6 \mathrm{~L} \mathrm{~min}^{-1}$, respectively. Results show 
that the count median diameters (CMDs) of the particles were $3.02 \pm 0.02,2.99 \pm 0.02$ and $3.02 \pm 0.02 \mu \mathrm{m}$, respectively, for voltage pulses of $14 \mathrm{~V}-18 \mu \mathrm{s}, 16 \mathrm{~V}-16 \mu \mathrm{s}$ and $18 \mathrm{~V}-14 \mu$ s with geometric standard deviations (GSDs) of 1.06. Assuming $d_{p}=3 \mu \mathrm{m}$ and $F_{v}=0.0216 \%$, the droplet diameter was calculated to be ideally close to $50 \mu \mathrm{m}$ which was the same size as the nozzle diameter. Since all particles were reduced in size by the same factor, the GSD of the droplet size distribution was the same as that of the dried particles. This demonstrates that the formation rate of droplets is controllable and the size of the formed droplets is uniform with the use of the inkjet generator. Unlike the inkjet generator, droplets produced for water-based solutions with a collision nebulizer are log-normally distributed with a CMD of about $0.9 \mu \mathrm{m}$ and a GSD of 1.8-2.0 (Moss and Cheng, 1995; Ulvecius et al., 1997; Hinds, 1999).

\section{Nebulization of PSL Suspension by Inkjet Aerosol \\ Generator}

An aerosol with only one sphere is normally referred to as a "singlet." When more than one sphere is present in a droplet, the resulting particle becomes a cluster or chain of spheres as the droplet dries. The probability $P(n)$ of $n$ spheres occurring in a droplet of diameter $d_{d}$ is given by (Hinds, 1999):

$$
P(n)=\frac{(\bar{x})^{n}}{n !} \exp (-\bar{x})
$$

where $\bar{x}$, the average number of spheres per droplet, is given by:

$$
\bar{x}=F_{v}\left(\frac{d_{d}}{d_{p}}\right)^{3}=C_{N} \times V_{d}
$$

where $F_{v}$ is the volume fraction of spheres of diameter $d_{p}$, $C_{N}$ is the number concentration of spheres in suspension and
$V_{d}$ is the volume of a droplet. In the present study, 3- $\mu \mathrm{m}$ PSL spheres were nebulized as a surrogate of microorganisms, such as yeast. As mentioned above, the size of droplets generated by the inkjet print head of $50-\mu \mathrm{m}$-diameter nozzle was $50 \mu \mathrm{m}$ in diameter. Based on theoretical calculation, Fig. 7 shows the fraction of singlets to droplets with solids, droplets with solids to total droplets and singlets to total droplets as a function of the number concentration of PSL suspensions. As the number concentration of PSL suspension increased, the ratio of singlets to droplets with solids decreased and droplets with solids to total droplets increased. As a result, the ratio of singlets to total droplets peaked as the number concentration of PSL suspension reached 1.53 $\times 10^{7} \mathrm{H} \mathrm{mL}^{-1}$.

Experimental results of the aerosol size distribution under different concentrations of PSL suspension are shown in Fig. 8. The test suspension was prepared from the original (highest concentration) PSL suspension in tenfold serial dilutions. A bimodal distribution was observed for all dilution ratios. The peak in small aerosol size represented the residue of droplets containing no PSL spheres, while another peak in large aerosol size illustrated the droplets with one or more PSL spheres. The average numbers of spheres per droplet were calculated to be $0.033,0.327$, and 3.27 for the corresponding PSL suspensions of $5 \times 10^{5}, 5 \times$ $10^{6}$, and $5 \times 10^{7} \# \mathrm{~mL}^{-1}$, respectively. Table 2 shows the fraction of droplets containing 0,1 or more spheres, when $d_{d}$ is $50 \mu \mathrm{m}$ and $d_{p}$ is $3 \mu \mathrm{m}$. The concentration of solute material, i.e., impurities, in PSL suspension was estimated to be $0.23 \%$. For the following bioaerosol survivability test, the yeast suspension was diluted with disinfected water to an approximate concentration of $5 \times 10^{6}$ cells $\mathrm{mL}^{-1}$ to yield challenging bioaerosols with more droplets containing only one yeast cell.

\section{Performance of an Inkjet Aerosol Generator in Yeast Survivability}

A piezoelectric inkjet print head as well as other types

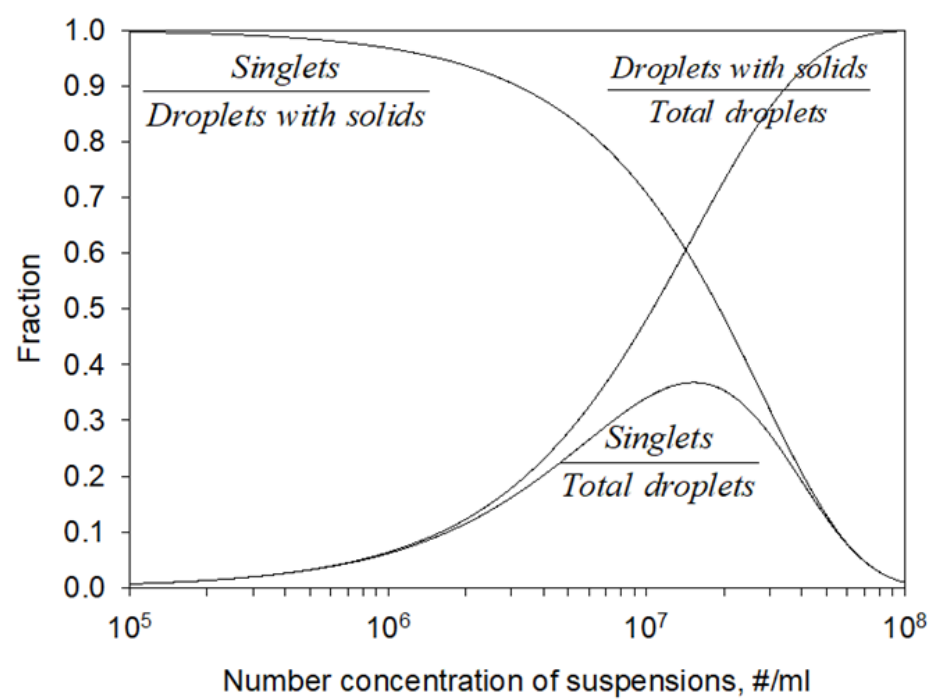

Fig. 7. Fraction of singlets to droplets with solids, droplets with solids to total droplets, and singlets to total droplets as a function of number concentration of PSL suspensions. 


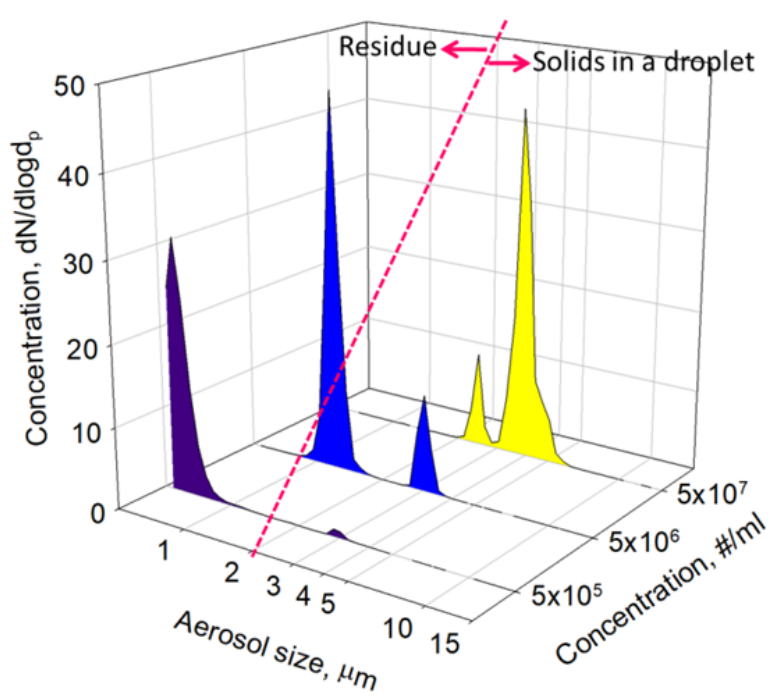

Fig. 8. Effect of PSL suspension concentration on the aerosol size distribution.

of nebulizers, including a Collison nebulizer and an ultrasonic nebulizer, were tested to rate the performance in yeast survivability. The relative survival rates with time for test nebulizers are shown in Fig. 9. Greatly outweighing the performance of the ultrasonic nebulizer, the inkjet generator exhibited excellent performance which is comparable to that of the Collison nebulizer in the preservation of yeast viability for prolonged nebulization. The use of an ultrasonic nebulizer is not recommended for bioaerosol survivability studies because the increasing temperature of the atomizing nozzle presented a detrimental effect to yeast viability as the yeast survival rate declined dramatically with the nebulization time. This finding confirms the capability of ultrasonic disinfection and is in good agreement with a previous study (Stamper et al., 2008). As for the Collison nebulizer, the viability of yeasts generated by a Collison nebulizer may gradually decrease with nebulization time due to the frequent recirculation of cell suspension with impaired yeast during prolonged nebulization.

\section{Application of an Inkjet Aerosol Generator for Bioaerosol Survivability Testing}

As mentioned above, the piezoelectric inkjet print head used in this study was shown to be controllable and reproducible for generating bioaerosols with excellent performance in microbial survivability with time. With a settling chamber, the inkjet print head system was employed to conduct bioaerosol survivability tests at controlled environmental conditions (for example, humidity) and exposure time, by sampling at different settling distances from the outlet of the aerosol generator.

When a yeast particle was introduced into the settling chamber, it underwent gravitational settling in still air and quickly reached its terminal settling velocity. Under this circumstance, the terminal settling velocity can be given by:

$$
V_{t s}=\frac{\rho_{p} d_{p}^{2} g}{18 \eta}
$$

where $\rho_{p}$ is the particle density, $d_{p}$ is the particle diameter,

Table 2. Fraction of droplets containing 0,1 and more spheres when $d_{d}$ is $50 \mu \mathrm{m}$ and $d_{p}$ is $3 \mu \mathrm{m}$.

\begin{tabular}{llll}
\hline Number concentration of particles & \multicolumn{3}{c}{ Fraction of droplets with indicated number of spheres } \\
\cline { 2 - 4 } in suspensions, $C_{N}$ & $\mathrm{n}=0$ & $\mathrm{n}=1$ & $\mathrm{n}>1$ \\
\hline $5 \times 10^{5}$ & 0.968 & 0.032 & 0.0005 \\
$5 \times 10^{6}$ & 0.721 & 0.236 & 0.043 \\
$5 \times 10^{7}$ & 0.038 & 0.124 & 0.838 \\
\hline
\end{tabular}

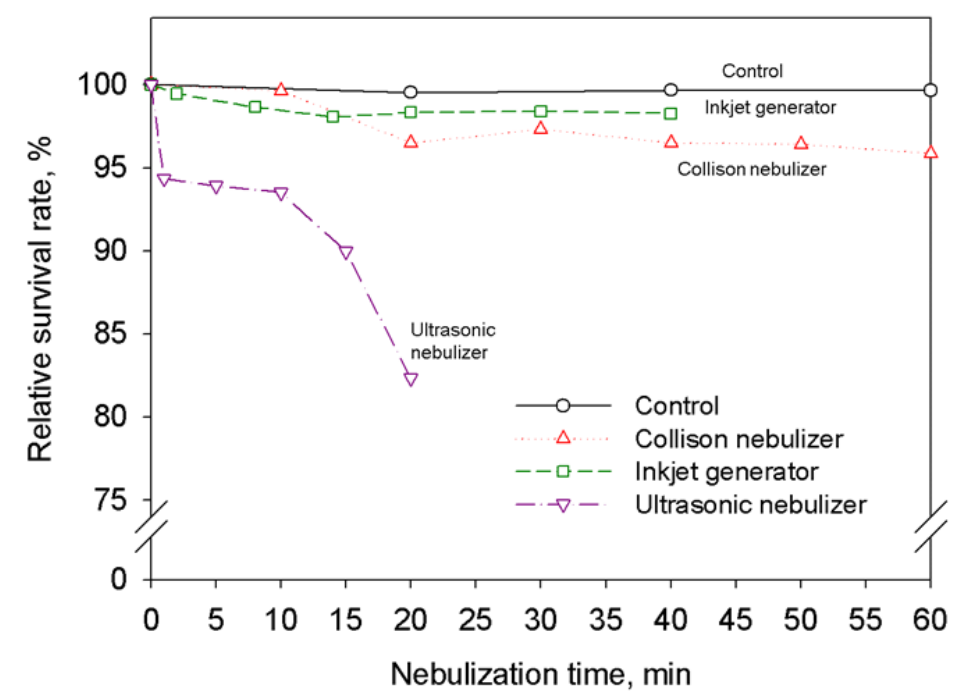

Fig. 9. Effect of nebulization methods on the yeast survivability. 
$g$ is the acceleration of gravity, and $\eta$ is the viscosity of the gas. In this case, the aerodynamic diameter of a yeast particle was $3 \mu \mathrm{m}$. The settling velocity of a yeast particle inside the chamber was calculated to be $0.027 \mathrm{~cm} \mathrm{~s}^{-1}$. The settling chamber was made of a 3-m vertical acrylic cylinder with an inner diameter of $20 \mathrm{~cm}$. A total air flow of $2 \mathrm{~L} \mathrm{~min}^{-1}$ was supplied to the chamber and achieved a downward wind speed of $0.106 \mathrm{~cm} \mathrm{~s}^{-1}$. Considering the settling velocity of a yeast particle and downward wind speed inside the chamber, a yeast particle could remain airborne in this $3-\mathrm{m}$ settling chamber for as long as 37.6 minutes.

The relative humidity $(\mathrm{RH})$ inside the settling chamber was adjusted to $20 \%$ and $80 \%$. By sampling at different distances from the outlet of inkjet generator, the inactivation kinetics of airborne yeast under the low and high humidity conditions were evaluated. Fig. 10 shows the inactivation kinetics of airborne yeast at $20 \%$ and $80 \%$ RH with the use of three types of bioaerosol samplers including a BioSampler, a gelatin filter cassette and a Marple impactor. The settling distances of 30,100, 200 and $300 \mathrm{~cm}$ correspond to the exposure times of $3.8,12.5,25.1$, and 37.6 minutes. The relative survival rates for samples taken by liquid impingement (a BioSampler) were in the ranges of 65$70 \%$, whereas those by impaction (a Marple sampler) and filtration (a gelatin filter cassette) were less than $20 \%$. Moreover, no significant differences in airborne yeast viability were found between low- and high-humidity conditions. The results imply that the bioaerosol sampling method might have a more significant effect on bioaerosol viability than that of the air humidity level.

During air sampling, the corresponding air velocities for the inlets of the three test samplers were 16,000, 240 and $6.8 \mathrm{~cm} \mathrm{~s}^{-1}$ for the BioSampler, Marple impactor and gelatin filter cassette, respectively. Regardless of the air velocity level, higher yeast survival rates were found for samples with the BioSamper (wet-collection) when compared to the other two samplers (dry-collection). The lowest yeast survival rate was found for samples with the Marple impactor and similar results were found by Zhen et al. (2013). This might be attributed to shear forces and surface impaction as well as desiccation caused by continuing air flow. In general, filtration units are not recommended for collecting viable aerosols due to the detrimental effect of desiccation during air sampling (Verreault et al., 2008). However, another study concluded that the high moisture content of gelatin filters caused minimum sampling stress (Zuo et al., 2014). Nevertheless, the experimental results of this study show that even with the use of high moisture content of gelatin filters, the filtration-based technique still causes detrimental effects on yeast survivability. For a better resolution of bioaerosol survivability, a wet collection method (for example, a BioSampler) is recommended for bioaerosol sampling in survivability studies to preserve the microbial viability.

With the use of an inkjet print head, a higher initial survival rate of challenging bioaerosols containing single microbial cell could be achieved, allowing better resolution in the evaluation of bioaerosol inactivation efficacy. Based on the experimental results, the optimal operating parameters for the inkjet generator to produce airborne singlet yeast cells were listed in Table 3.

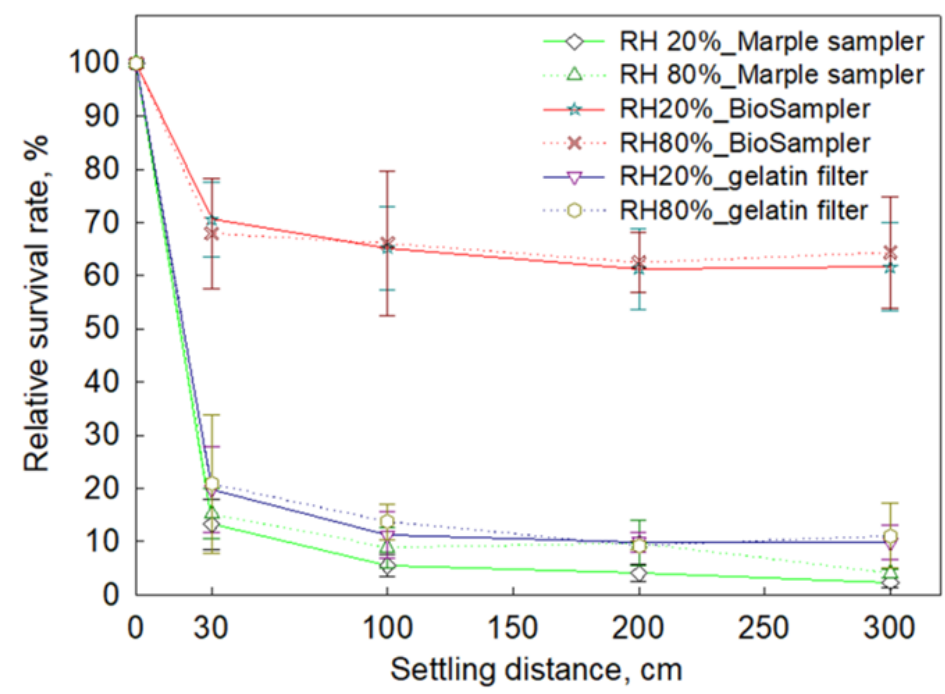

Fig. 10. Relative survival rates of airborne yeast with different exposure times in terms of settling distance at $20 \%$ and $80 \% \mathrm{RH}$ with the use of three samplers.

Table 3. The optimal operating parameters for the inkjet generator to produce singlets.

\begin{tabular}{ll}
\hline Parameter & Optimal value or range \\
\hline Excitation voltage \& dwell time & The shaded area in Fig. 3 \\
Dispersion air flow rate, $\mathrm{L} \mathrm{min}^{-1}$ & 0.4 \\
Yeast suspension concentration, cells mL & $5 \times 10^{6}$ \\
\hline
\end{tabular}




\section{CONCLUSIONS}

This study successfully demonstrated the feasibility and the advantages of using a piezoelectric inkjet print head to generate viable aerosols. Applying a square wave with appropriate combinations of voltage and pulse duration enabled the inkjet system to produce a liquid with rheological properties similar to those of water, and the particle generation rate was manipulated by adjusting the pulse frequency. Dispersion air was required to reduce coagulation. The generated droplets were approximately $50 \mu \mathrm{m}$ in size with a GSD of 1.06. The size distribution of the particles dried from 3- $\mu \mathrm{m}$ PSL suspensions revealed two peaks, one for the smaller size range and another for the larger size range, which represented the soluble residue of the suspension, and dried particles from the main droplets, respectively. The greater the volume fraction of the solid particles, the fewer empty droplets were formed. However, an excessive volume fraction of solid particles reduced the probability of droplets containing only one solid particle. The appropriate concentration for the $3-\mu \mathrm{m}$ solid suspension was on the scale of $10^{6} \# \mathrm{~mL}^{-1}$. The fraction of droplets containing at least one particle was 0.26 , whereas the fraction of droplets containing only one particle was 0.86 .

\section{ACKNOWLEDGMENTS}

This research was financially supported by the Ministry of Science and Technology, Taiwan, R. O. C., under the grants: MOST-106-3011-F-002-001 and MOST-104-2221E-002-031-MY3.

\section{REFERENCES}

Agranovski, I.E., Safatov, A.S., Pyankov, O.V., Sergeev, A.A., Sergeev, A.N. and Grinshpun, S.A. (2005). Longterm sampling of viable airborne viruses. Aerosol Sci. Technol. 39: 912-918.

Dougherty, G.M., Hadley, D.R., O'Connor, P.R. and Bottiger, J.R. (2007). Engineered aerosol production for laboratory scale chemical/biological test and evaluation, Report No. UCRL-TR-230829. Lawrence Livermore National Laboratory (US), Livermore, CA, USA.

Ferris, C.J., Gilmore, K.J., Beirne, S., McCallum, D., Wallace, G.G. and Het Panhuis, M. (2013). Bio-ink for inkjet printing of living cells. Nanotechnology 2013: Electronics, Devices, Fabrication, MEMS, Fluidics and Computational - 2013 NSTI Nanotechnology Conference and Expo, NSTI-Nanotech 2013, May 12, 2013-May 16, 2013, Washington, DC, USA.

Gañán-Calvo, A.M. and Barrero, A. (1999). A novel pneumatic technique to generate steady capillary microjets. J. Aerosol Sci. 30: 117-125.

Hinds, W.C. (1999). Aerosol technology: Properties, behavior, and measurement of airborne particles (2nd ed.). Wiley-Interscience, New York (Chapter 21).

Huang, J., Cai, R. and Zhang, K. (2012). Experiments and analysis of drop on demand cell printing. Res. J. Appl.
Sci. Eng. Technol. 4: 93-96.

Iida, K., Sakurai, H., Saito, K. and Ehara, K. (2014). Inkjet aerosol generator as monodisperse particle number standard. Aerosol Sci. Technol. 48: 789-802.

Kreyling, W.G. and Ferron, G.A. (1982). Aerosol nebulizer for low concentrated particle suspensions. J. Aerosol Sci. 14: 264-267.

Kwon, K.S. (2009). Waveform design methods for piezo inkjet dispensers based on measured meniscus motion. J. Microelectromech. Syst. 18: 1118-1125.

Kwon, K.S. (2010). Experimental analysis of waveform effects on satellite and ligament behavior via in situ measurement of the drop-on-demand drop formation curve and the instantaneous jetting speed curve. $J$. Micromech. Microeng. 20: 115005.

Lahde, A., Raula, J., Kauppinen, E.I., Watanabe, W., Ahonen, P.P. and Brown, D.P. (2006). Aerosol synthesis of inhalation particles via a droplet-to-particle method. Part. Sci. Technol. 24: 71-84.

Lever, M.S., Williams, A. and Bennett, A.M. (2000). Survival of mycobacterial species in aerosols generated from artificial saliva. Lett. Appl. Microbiol. 31: 238-241.

Lin, C.Y., Meng, H.C. and Fu, C. (2011). An ultrasonic aerosol therapy nebulizer using electroformed palladiumnickel alloy nozzle plates. Sens. Actuators, A 169: 187193.

Lin, H.J. (2006). The effects of operating parameters on micro-droplet formation in a piezoelectric inkjet printhead using a double pulse voltage pattern. Mater. Trans. 47: 375-382.

Liu, Y.F., Tsai, M.H., Pai, Y.F. and Hwang, W.S. (2013). Control of droplet formation by operating waveform for inks with various viscosities in piezoelectric inkjet printing. Appl. Phys. A 111: 509-516.

Mainelis, G., Berry, D., Reoun An, H., Yao, M., DeVoe, K., Fennell, D.E. and Jaeger, R. (2005). Design and performance of a single-pass bubbling bioaerosol generator. Atmos. Environ. 39: 3521-3533.

May, K.R. (1973). The collison nebulizer: Description, performance and application. J. Aerosol Sci. 4: 235-243.

Meier, R. (2006). Sampling microbes in compressed gases. Cleanroom Technol. 12: 14-15.

Moss, O.R. and Cheng, Y.S. (1995). Generation and characterization of test atmospheres: Particles and droplets. In Concepts in inhalation toxicology, 2nd edition, McClellan, R. and Henderson, R. (Eds.), Taylor \& Francis, Washington, DC, USA.

Nikander, K., Turpeinen, M. and Wollmer, P. (1999). The conventional ultrasonic nebulizer proved inefficient in nebulizing a suspension. J. Aerosol Med. 12: 47-53.

Parsa, S., Gupta, M., Loizeau, F. and Cheung, K.C. (2010). Effects of surfactant and gentle agitation on inkjet dispensing of living cells. Biofabrication 2: 25003.

Porstendörfer, J., Gebhart, J. and Röbig, G. (1977). Effect of evaporation on the size distribution of nebulized aerosols. J. Aerosol Sci. 8: 371-380.

Reis, N., Ainsley, C. and Derby, B. (2005). Ink-jet delivery of particle suspensions by piezoelectric droplet ejectors. J. Appl. Phys. 97: 4903-4906. 
Reponen, T., Willeke, K., Ulevicius, V., Grinshpun, S.A. and Donnelly, J. (1997). Techniques for dispersion of microorganisms into air. Aerosol Sci. Technol. 27: 405421.

Roux, J.M., Kaspari, O., Heinrich, R., Hanschmann, N. and Grunow, R. (2013). Investigation of a new electrostatic sampler for concentrating biological and non-biological aerosol particles. Aerosol Sci. Technol. 47: 463-471.

Saunders, R.E., Gough, J.E. and Derby, B. (2008). Delivery of human fibroblast cells by piezoelectric dropon-demand inkjet printing. Biomaterials 29: 193-203.

Shih, W.J., Jeong, Y.S., Choi, K. and Shin, W.G. (2015). The effect of inkjet operating parameters on the size control of aerosol particles. Aerosol Sci. Technol. 49: 1256-1262.

Shimoda, T., Morii, K., Seki, S. and Kiguchi, H. (2003). Inkjet printing of light-emitting polymer displays. $M R S$ Bull. 28: 821-827.

Stamper, D.M., Holm, E.R. and Brizzolara, R.A. (2008). Exposure times and energy densities for ultrasonic disinfection of Escherichia coli, Pseudomonas aeruginosa, Enterococcus avium, and sewage. J. Environ. Eng. Sci. 7: 139-146.

Sung, G., Kim, H.U., Shin, D., Shin, W.G. and Kim, T. (2018). High Efficiency Axial Wet Cyclone Air Sampler. Aerosol Air Qual. Res. 18: 2529-2537.

Taylor, K.M.G. and McCallion, O.N.M. (1997). Ultrasonic nebulisers for pulmonary drug delivery. Int. J. Pharm. 153: 93-104.

Terzano, C. and Allegra, L. (2002). Importance of drug delivery system in steroid aerosol therapy via nebulizer. Pulm. Pharmacol. Ther. 15: 449-454.

The, R., Yamaguchi, S., Ueno, A., Akiyama, Y. and Morishima, K. (2013). Piezoelectric inkjet-based one cell per one droplet automatic printing by image processing. 2013 26th IEEE/RSJ International Conference on Intelligent Robots and Systems: New Horizon, IROS 2013, November 3, 2013-November 8, 2013, Tokyo, Japan.

Thomas, R.J., Webber, D., Sellors, W., Collinge, A., Frost, A., Stagg, A.J., Bailey, S.C., Jayasekera, P.N., Taylor, R.R., Eley, S. and Titball, R.W. (2009). Generation of large droplet aerosols within microbiological containment using a novel flow-focussing technique. Aerobiologia 25: 75-84.

Thomas, R.J., Webber, D., Hopkins, R., Frost, A., Laws, T., Jayasekera, P.N. and Atkins, T. (2011). The cell membrane as a major site of damage during aerosolization of Escherichia coli. Appl. Environ. Microbiol. 77: 920-925. Tolchinsky, A.D., Sigaev, V.I., Varfolomeev, A.N., Uspenskaya, S.N., Cheng, Y.S. and Su, W.C. (2011). Performance evaluation of two personal bioaerosol samplers. J. Environ. Sci. Health, Part A 46:1690-1698. Tsai, C.S., Mao, R.W., Lin, S.K., Zhu, Y., Tsai, S.C., Brenner, M., Mahon, S., Mukai, D. and Boss, G. (2013). Pocket-sized ultrasonic nebulizer for inhalation drug delivery. 2013 IEEE International Ultrasonics Symposium, IUS 2013, July 21, 2013-July 25, 2013, Prague, Czech Republic.

Ulvecius, V., Willke, K., Grinshpun, S., Donnelly, J., Lin, X. and Mainelis, G. (1997). Aerosolization of particles from a bubbling liquid: Characteristics and generator development. Aerosol Sci. Technol. 26: 175-190.

Verreault, D., Moineau, S. and Duchaine, C. (2008). Methods for sampling of airborne viruses. Microbiol. Mol. Biol. Rev. 72: 413-444.

Watts, A.B., McConville, J.T. and Williams, R.O. (2008). Current therapies and technological advances in aqueous aerosol drug delivery. Drug Dev. Ind. Pharm. 34: 913922.

Woo, M.H., Grippin, A., Anwar, D., Smith, T., Wu, C.Y. and Wander, J.D. (2012). Effects of relative humidity and spraying meium on UV decontamination of filters loaded with viral aerosols. Appl. Environ. Microbiol. 78: 5781-5787.

Wu, Y. and Yao, M. (2010). Inactivation of bacteria and fungus aerosols using microwave irradiation. J. Aerosol Sci. 41: 682-693.

Yamaguchi, S., Ueno, A., Akiyama, Y. and Morishima, K. (2012a). Cell patterning through inkjet printing of one cell per droplet. Biofabrication 4: 045005.

Yamaguchi, S., Ueno, A. and Morishima, K. (2012b). Stable ejection of micro droplets containing microbeads by a piezoelectric inkjet head. J. Micro-Nano Mechatron. 7: 87-95.

Zhen, H., Han, T., Fennell, D.E. and Mainelis, G. (2013). Release of free DNA by membrane-impaired bacterial aerosols due to aerosolization and air sampling. Appl. Environ. Microbiol. 79: 7780-7789.

Zhen, H., Han, T., Fennell, D.E. and Mainelis, G. (2014). A systematic comparison of four bioaerosol generators: Affect on culturability and cell membrane integrity when aerosolizing Escherichia coli bacteria. J. Aerosol Sci. 70: 67-79.

Zuo, Z., Kuehn, T.H., Bekele, A.Z., Mor, S.K., Verma, H., Goyal, S.M., Raynor, P.C. and Pui, D.Y.H. (2014). Survival of airborne MS2 bacteriophage generated from human saliva, artificial saliva, and cell culture medium. Appl. Environ. Microbiol. 80: 2796-2803.

Received for review, July 7, 2018 Revised, November 9, 2018 Accepted, December 19, 2018 